\title{
In-vessel saddle coils for MHD control in ASDEX Upgrade
}

\author{
W. Suttrop ${ }^{\text {a }}$, O. Gruber ${ }^{\text {a }}$, S. Günter ${ }^{\text {a }}$, D. Hahn ${ }^{\text {a }}$, A. Herrmann ${ }^{\text {a }}$, \\ M. Rott ${ }^{\text {a }}$, T. Vierle ${ }^{\text {a }}$, U. Seidel ${ }^{\text {a }}$, M. Sempf ${ }^{\text {a }}$, B. Streibl ${ }^{\text {a }}$, \\ E. Strumberger ${ }^{a}$, D. Yadikin ${ }^{a}$, O. Neubauer ${ }^{b}$, B. Unterberg ${ }^{b}$, \\ E. Gaio ${ }^{\mathrm{c}}$, V. Toigo ${ }^{\mathrm{c}}$, P. Brunsell ${ }^{\mathrm{d}}$, and the ASDEX Upgrade Team \\ a Max-Planck-Institut für Plasmaphysik, EURATOM Association, D-85740 Garching, \\ Germany \\ b Institut für Energieforschung - Plasmaphysik, Forschungszentrum Jülich GmbH, \\ Association EURATOM/FZJ, Trilateral Euregio Cluster, D-52425 Jülich, Germany \\ ${ }^{\mathrm{c} C o n s o r z i o ~ R F X, ~ A s s o c i a z i o n e ~ E U R A T O M-E N E A, ~ I-35127 ~ P a d o v a ~}$ \\ ${ }^{\mathrm{d}}$ Royal Institute of Technology KTH, Association EURATOM-VR, SE-10044 Stockholm
}

\begin{abstract}
A set of 24 in-vessel saddle coils is planned for MHD control experiments in ASDEX Upgrade. These coils can produce static and alternating error fields for suppression of Edge Localised Modes, locked mode rotation control and, together with additional conducting wall elements, resistive wall mode excitation and feedback stabilisation experiments. All of these applications address critical physics issues for the operation of ITER. This extension is implemented in several stages, starting with two poloidally separated rings of eight toroidally distributed saddle coils above and below the outer midplane. In stages 2 and 3, eight midplane coils around the large vessel access ports and 12 AC power converters are added, respectively. Finally (stage 4), the existing passive stabilising loop (PSL), a passive conductor for vertical growth rate reduction, will be complemented by wall elements that allow helical current patterns to reduce the RWM growth rate for active control within the accessible bandwidth. The system is capable of producing error fields with toroidal mode number $n=4$ for plasma edge ergodisation with core island width well below the neoclassical tearing mode seed island width even without rotational shielding. Phase variation between the three toroidal coil rings allows to create or avoid resonances with the plasma safety factor profile, in order to test the importance of resonances for ELM suppression.
\end{abstract}

Key words: Tokamaks, ASDEX Upgrade, MHD control, Edge Localised Modes, Locked Mode, Resistive Wall Mode

PACS: 52.55 Fa, 07.05.Fb, 07.55.Db 


\section{Introduction}

Performance improvement in ITER and a fusion reactor over expected parameters obtained by extrapolation from present-day tokamaks will likely require active control of MHD instabilities. The energy loss associated with Edge Localised Modes (ELMs) in ITER might exceed the tolerable limit of $\Delta W_{E L M} \approx 10 \mathrm{MJ}$ to avoid evaporation of $\mathrm{C}$ or $\mathrm{W}$ wall [1]. The scaling of ELM loss energy is not known, but present data suggests an unfavourable dependence on pedestal collisionality $v^{*}[2]$. Techniques to avoid large ELMs are therefore being investigated, for example ELM loss reduction by injection of cryogenic pellets [3], small ELM regimes [4-6] and stationary ELM-free regimes [7,8]. It has been observed early on in COMPASS-D that non-axisymmetric error fields can reduce the size of ELMs [9]. More recently, ELM mitigation is studied in DIII-D with more edge-localised $n=3$ magnetic perturbations produced by a set of $2 \times 6$ in-vessel saddle coils [10]. Complete ELM suppression is obtained at low $v^{*}$ if a helical perturbation field of sufficient strength is applied which matches the plasma safety factor $q$ ("resonant magnetic perturbation"). In a first interpretation [11], ELM suppression is attributed to ergodisation of the magnetic field in the pedestal region, causing additional radial heat transport and, for fixed heat source, a reduction of the pressure gradient to values below the MHD stability limit encountered at the ELM onset. Many open questions remain. In several experiments, application of a non-axisymmetric perturbation field is accompanied with a reduction of plasma density while the temperature is unchanged or even increases (e.g. [11]). The role of the resonance for complete ELM suppression is not clear. Because of the additional loss channel due to parallel transport one would expect that for sub-threshold error fields the ELM frequency is reduced, which is not observed.

It is hence warranted to study the physics of ELM suppression with large configuration flexibility in detail in existing tokamaks. Here we present an overview of a system for MHD control with active saddle coils in ASDEX Upgrade. These coils offer large configuration flexibility for ELM suppression experiments and AC capability for feedback-suppression of resistive wall modes (RWM) and rotation control of neoclassical tearing modes (NTM, not discussed here). Here, suitability of the proposed coils for physics investigations is discussed. We focus on ELM suppression experiments and briefly discuss the suitability for RWM stabilisation. The mechanical layout of the coils and design verification is presented in [12]. Electromagnetic modeling of the coils performance is described in [13].

Email address: Wolfgang. Suttrop@ipp.mpg.de (W. Suttrop). 


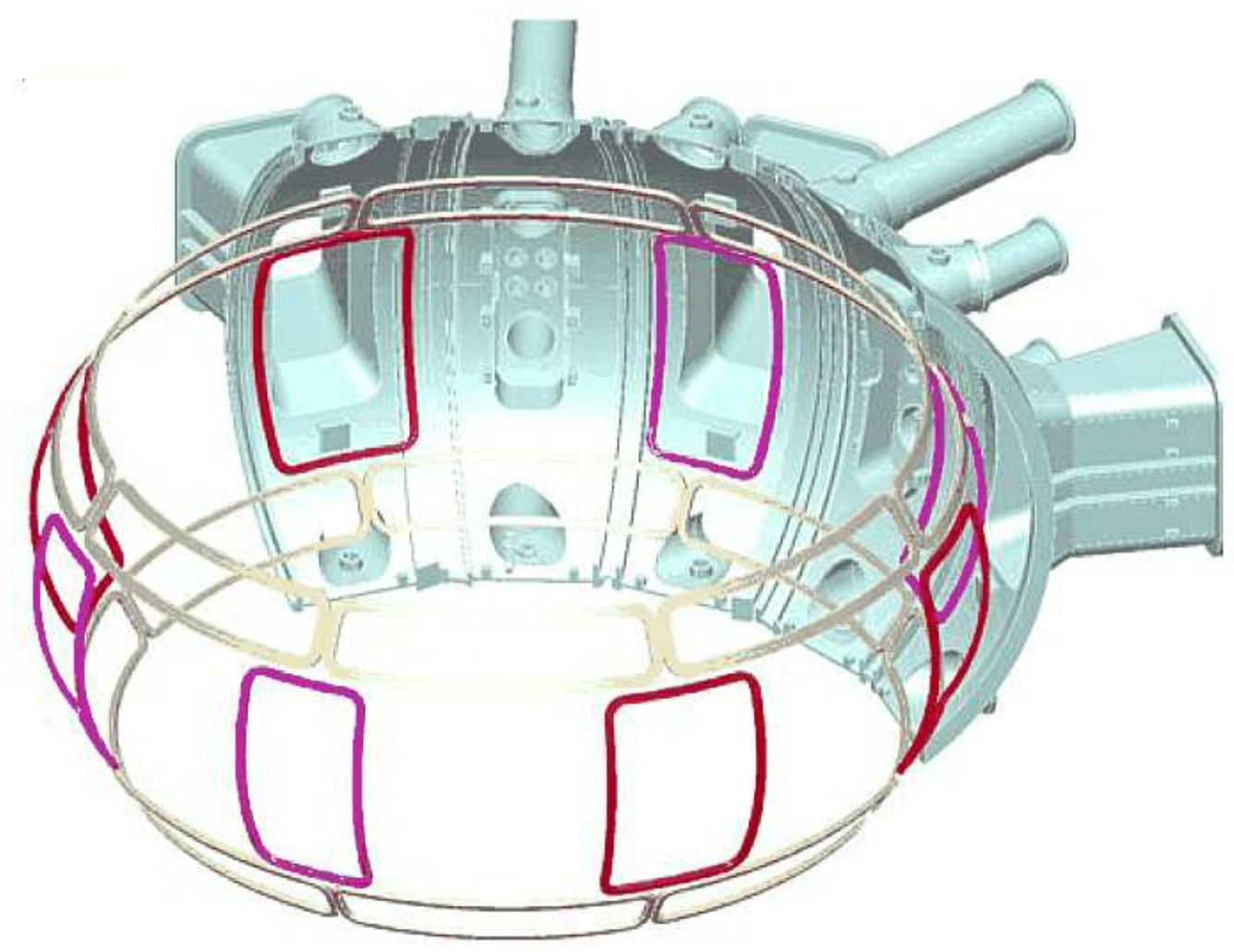

Fig. 1. 3D view of active in-vessel coils

\section{Design and properties of the saddle coil set}

The proposed coil set consists of 24 saddle coils mounted inside the vacuum vessel of ASDEX Upgrade as shown schematically in Fig. 1. The coils are placed at the low field side and close to the plasma for fast response and high flexibility of the structure of the field produced. The enhancement will be carried out in several stages. First, 8 upper and 8 lower coils (dubbed Bu-coils and Bl-coils, respectively) will be installed which allow for DC and AC operation up to a frequency of $f_{\max }=$ $1.2 \mathrm{kHz}$. The coils can be operated in series with an existing single power supply for ELM control experiments with toroidal mode numbers $n=1, n=2$, or $n=4$, or can be supplied by individual power supplies for more flexible field configurations.

As a second stage, it is planned to complement this set with eight additional coils around midplane ports (A-coils). Due to space restrictions these coils cannot span the toroidal circumference of the torus. Also, because of restrictions set by the installed heating systems, there are two different types of coils with different toroidal width, $12^{0}$ and $19.3^{\circ}$ wide, respectively. These coils are more distant from passive conductors (PSL and future conducting wall elements) and are designed for $f_{\max }=3 \mathrm{kHz}$ for locked mode avoidance. For $n=4$, toroidally neighbouring coils carry currents of opposite polarity. The upper and lower toroidal rings can have a $90^{\circ}$ phase shift in clock-wise or anti-clock wise direction, leading to four different 


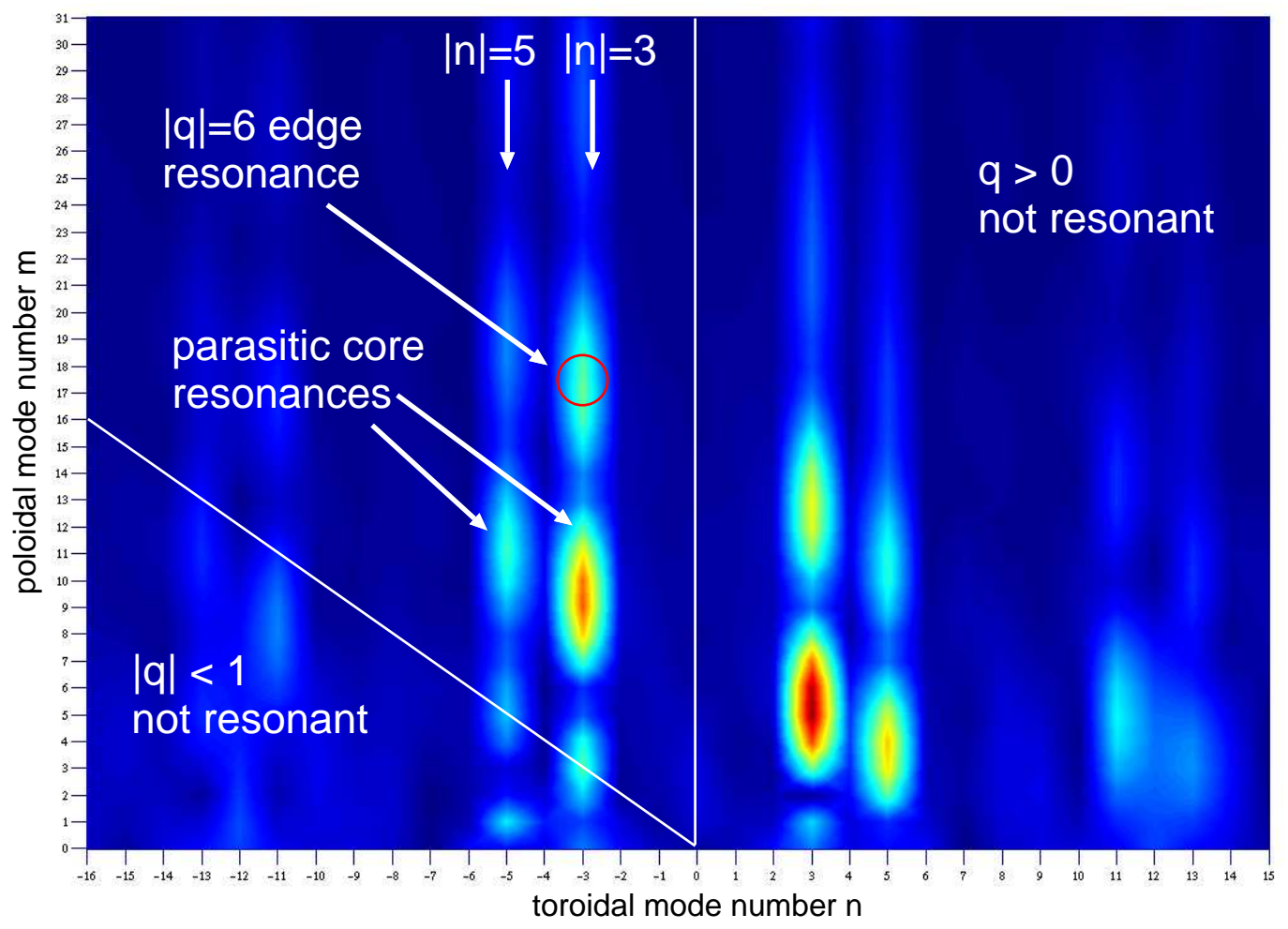

Fig. 2. Mode number $m, n$ spectrum perturbation (best fit to $n=-3$ at the $q=-6$ surface)

parity configurations.

In a third step, 12 independent AC power supplies are installed, each of which can supply two coils in series. For example, toroidally opposite coils can be connected in series or anti-series, resulting in perturbations with all even or all odd toroidal mode numbers $n$, respectively. Also, perturbations with $n \leq 3$ can be toroidally rotated with a quasi-continuous phase, analogous to three phase current. This feature allows to improve diagnosis of the non-axisymmetric perturbed plasma with the existing sightlines by varying the toroidal phase, and hence achieve quasi $2 \mathrm{D}$ resolution of the existing measurements similar to measurements obtained with the TEXTOR Dynamic Ergodic Divertor [14]. Finally, the phase between Bu and A coils, and between BI and A coils can be varied independently. This allows to switch between resonant or non-resonant conditions without changing the plasma safety factor $q$.

Fig. 2 shows the $m, n$ mode spectrum (2D Fourier transform) of the normal vacuum field produced by the saddle coils for a best fit to a $n=-3$ perturbation at the $q=-6$ surface in a typical ASDEX Upgrade H-mode plasma with monotonous $q$ profile, in this case with $q_{0} \approx 1.0$ and $q_{95}=5$. Only the $m>0$ half plane is shown. Modes with $n<0$ and $|q|>1$ are resonant in the plasma, $n>0$ corresponds to opposite helical orientation of plasma field and perturbation. The spectrum is asymmetric in $n$ because the best fitting field has neither odd nor even toroidal parity. In addition to the desired $n=3$ mode, the finite number of toroidally dis- 
tributed saddle coils leads to spatial aliasing, thus producing an $n=5$ sideband. The finite toroidal extent of each coil causes deviations from a sinusoidal field variation, thereby creating higher $n$ harmonics. The poloidal spectrum $(m)$ is affected by the concentration of the coils at the low field side. Apart from the desired resonance at $m=18$, there is a stronger, parasitic, resonance at $m=9(q=3)$. It should be stressed that spectral imperfections like those described above are unavoidable with this type of saddle coils. For an elongated and diverted tokamak plasma a full poloidal coverage (for a clean $m$ spectrum) is impractical because of the extremely high multipole moments required near the top and bottom of the plasma.

In a fourth stage, a conducting shell is added on the low field side, in between the existing upper and lower passive stabilising loops (PSL). While the PSL alone allows for induced currents that produce a radial field to reduce the vertical growth rate, the PSL branches and the new shell connected together allow helical currents. A feedback system consisting of distributed poloidal field sensors and a digital controller for $n=1 \mathrm{RWM}$ control are envisaged at this stage.

Finally, with optional 24 independent AC power supplies, odd and even $n$ perturbations can be made simultaneously, and the full voltage swing (maximum frequency) is available for each coil. This allows for multi-mode RWM control $(n=1,2, \ldots)$ and, simultaneously, high $n$ DC components for ELM suppression in a combined high performance plasma scenario.

\section{Resonant Magnetic Perturbation for ELM suppression}

The objectives of investigating the effect of error fields on ELMs in ASDEX Upgrade are to test the role of a resonance between external field and $q$ profile, study edge transport and stability for varying degrees of edge ergodisation (which implies diagnosis of the H-mode edge pedestal and ELM behaviour) and investigate different perturbation field configurations that may be useful in ITER.

The degree of ergodisation is described by the "Chirikov parameter" [15], $\sigma=$ $\left(\psi_{\max }-\psi_{\min }\right) /\left(\psi_{q_{\text {in }}}-\psi_{q_{\text {out }}}\right)$, which is defined here as the radial field line excursion in terms of the minimum and maximum unperturbed poloidal flux, normalised by the flux difference between nearest neighbor rational surfaces $q_{\text {in }}=m_{\text {in }} / n, q_{\text {out }}=$ $m_{\text {out }} / n$, where $m_{\text {out }}=m_{\text {in }}+1$ is an integer and $n$ is the fundamental toroidal mode number of the applied perturbation field. Ergodisation, or field line diffusion, is recognised by the transition from purely oscillatory into growing radial excursion. This is seen in puncture (Poincaré) plots of field lines in a poloidal plane (shrinking islands and appearance of space-filling field lines) and by a non-zero Lyapunov exponent that describes $\psi(\Phi)(\Phi$ : toroidal angle) for an individual field line. We find as a practical threshold for edge ergodisation, $\sigma_{c r i t}=1$, which corresponds to the picture of onset of radial "overlap" of magnetic island chains created by the 


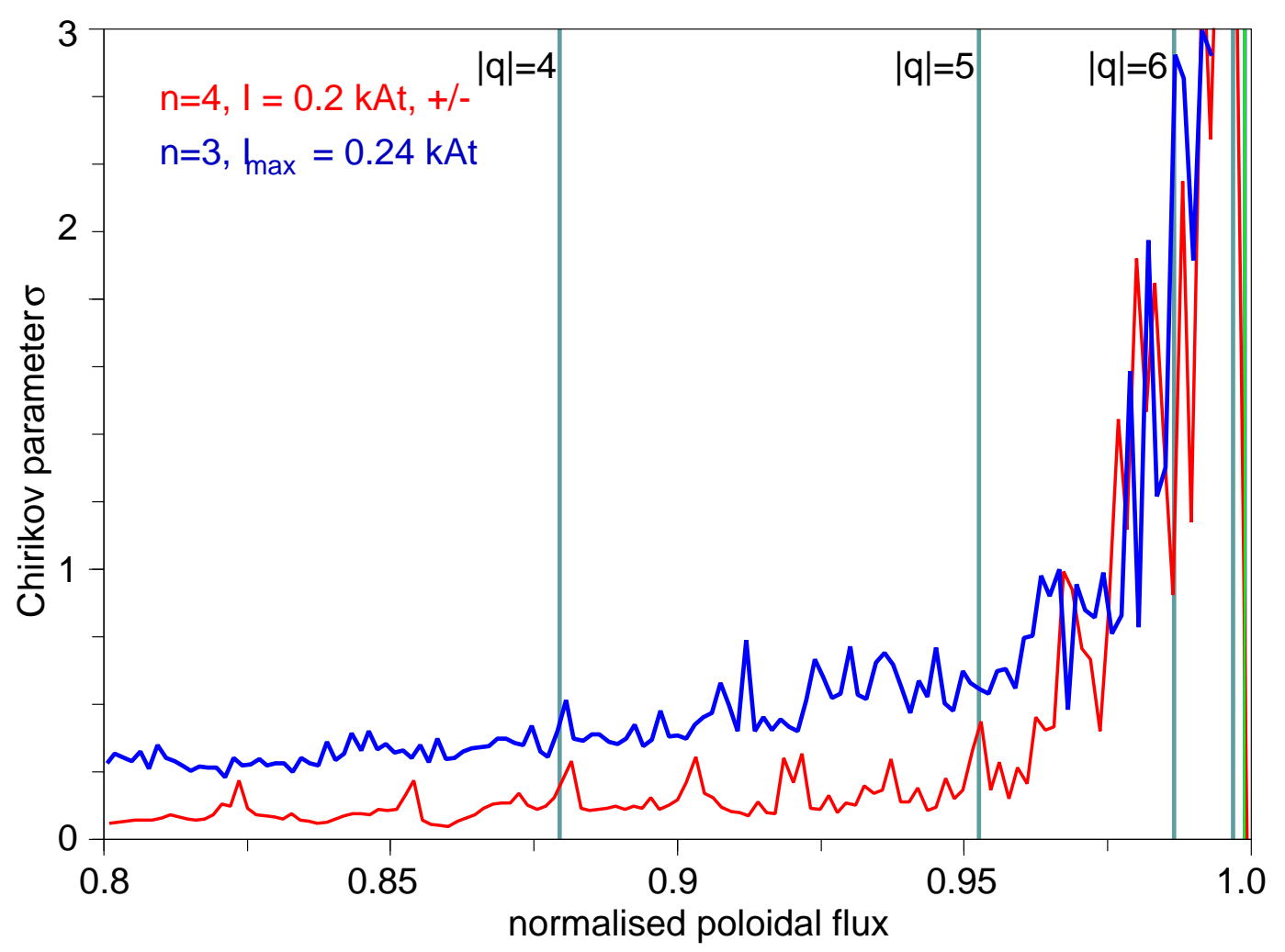

Fig. 3. Chirikov parameter $\sigma$ for $n=3$ and $n=4$ perturbations. The coil current is adjusted for slight ergodisation $(\sigma=1 \ldots 3)$ in the edge barrier region $\rho>0.95$

perturbation. With increasing perturbation field, ergodisation sets in always at the edge, in the strong magnetic shear region.

Fig. 3 shows $\sigma$ as a function of normalised flux for an $n=3$ and an $n=4$ external field. The coil current (maximum current for $n=3$ ) is chosen to obtain $\sigma \approx 3$ and turns out to be similar in both cases $\left(I_{\text {coil }}=0.24 \mathrm{kA} \times\right.$ turns $(\mathrm{kAt}) \max$. for $n=3$, and $0.2 \mathrm{kAt}$ for $n=4$ ). The field line excursion in cm (outboard midplane) is shown in Fig. 4. For the same pedestal $\sigma$, the core excursions are much smaller for $n=4$ than for $n=3$. The island width ( $2 \times$ max. radial excursion) has to be compared with a typical NTM seed island width of $1 \mathrm{~cm}$. Rotational shielding will reduce the perturbation field in the plasma core, but will not much affect the ergodisation in the edge pedestal region. The present saddle coil design allows up to $I_{\text {coil }}=5 \mathrm{kAt}$, providing a factor of 20 headroom to compensate for shielding of the perturbation field.

The availability of three coil subsets at different poloidal positions allows to influence the poloidal $(\mathrm{m})$ spectrum of the perturbation. Figure 5 shows the poloidal spectrum for $n=4$ perturbations with two different phasings of $\mathrm{Bu}-, \mathrm{Bl}-$ and $\mathrm{A}-$ coils (indicated in the inserts) as a function of normalised poloidal flux radius $\Psi$. The resonant $m=q(\Psi) / n$ for ASDEX Upgrade shot \#17151 at $t=3.85 \mathrm{~s}$ is overlaid. The strong parasitic low $m$ perturbation is visible at all radii. Depending on 


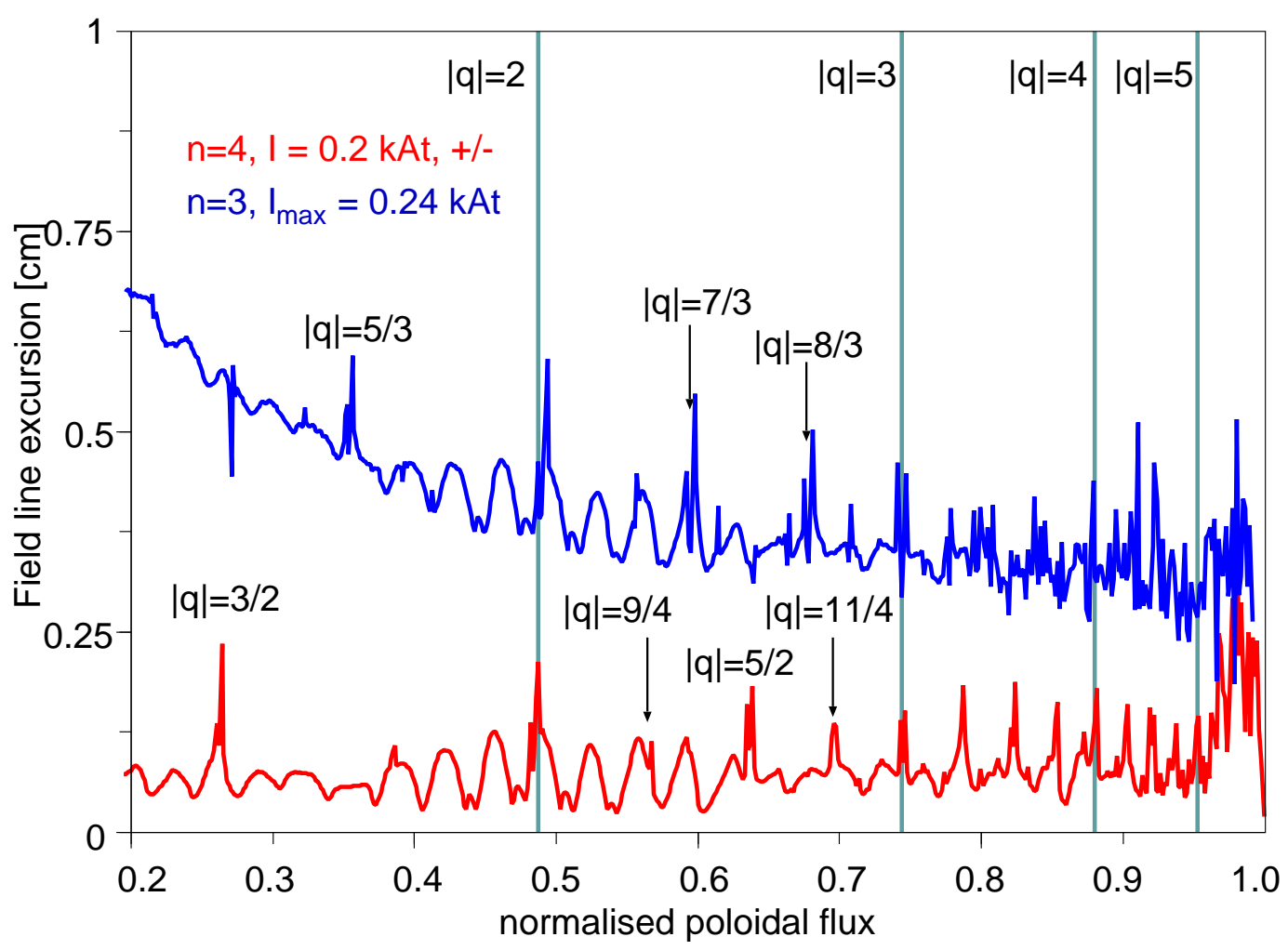

Fig. 4. Field line excursion for the cases of Fig. 3 showing that for $n=4$ the parasitic core island width is smaller than for $n=3$.

the phasing, a resonant field component with the plasma $q$ profile is obtained ('+/-' phasing) or avoided ('-/-' phasing).

\section{RWM control}

The active coils can also be used for feedback stabilisation of the resistive wall mode (RWM). Since in ASDEX Upgrade the vessel wall is relatively distant from the plasma, is it planned to extend the PSL with copper wall elements which cover the area between the upper and lower PSL branch. Relatively large holes are needed in some sectors for heating and diagnostics systems, leading to a non-axisymmetric, multiply connected wall. As a consequence, the eigenvalues corresponding to a pair of unstable RWMs are no longer degenerate. Due to the non-axisymmetric wall, the wall current patterns induced by the two modes are different, leading to different current decay times and hence different RWM growth rates. Furthermore, different toroidal mode numbers couple, i.e. eigenmodes are no longer characterised by a single toroidal mode number $n$.

These three-dimensional effects are treated by the full 3D stability codes STARWALL (ideal and resistive wall stability) [16,17] and OPTIM (stability of closed loop system, optimisation of feedback gains) [18]. The proposed coil set and the finite ele- 

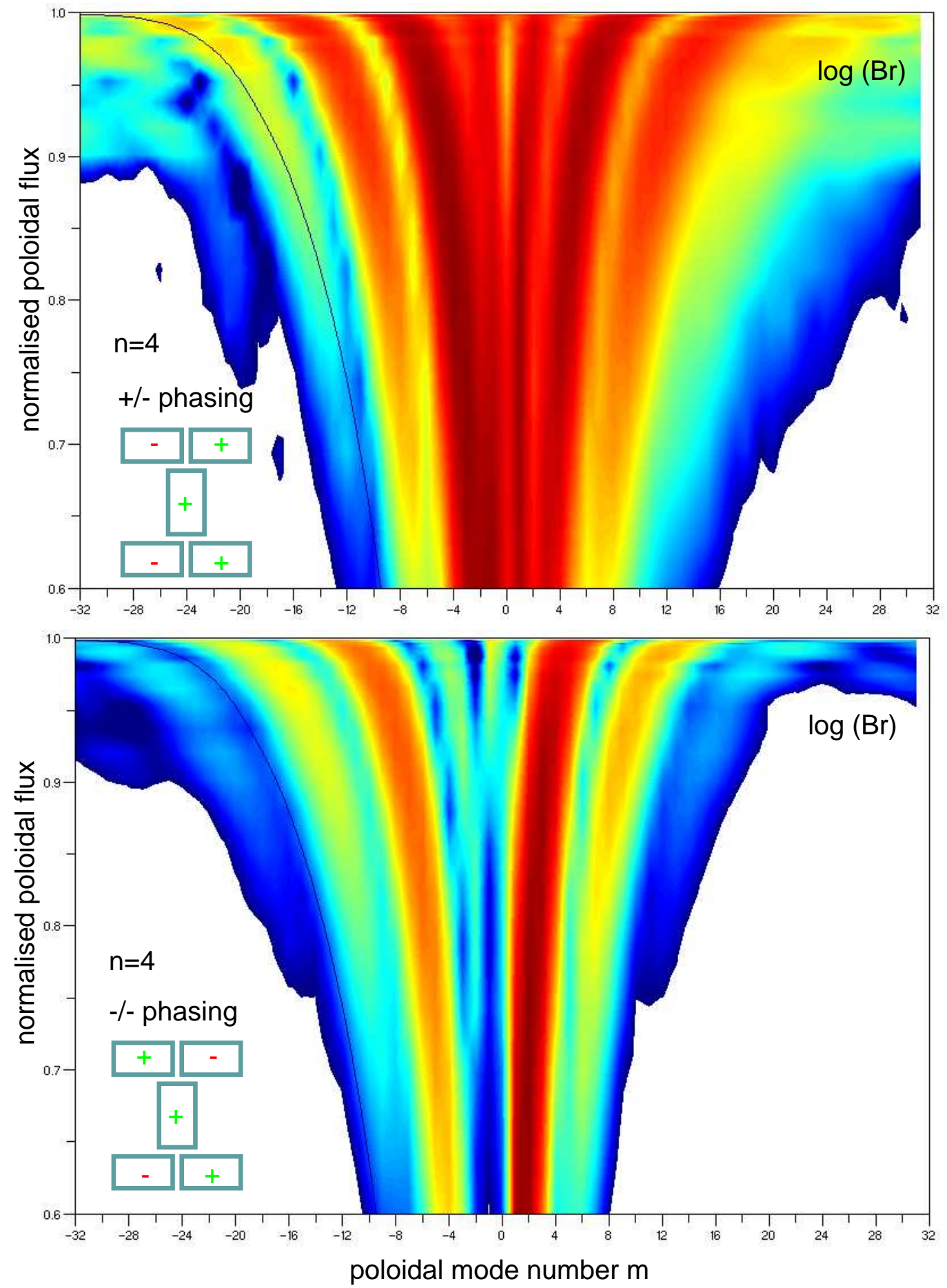

Fig. 5. Poloidal mode spectrum as a function of radius for an $n=4$ perturbation, plus-minus phasing (top) and minus-minus phasing (bottom). The black line denotes the $q$ profile of AUG discharge 17151 at $t=3.85 \mathrm{~s}$.

ment wall model shown in Fig. 6 are used to assess the effectiveness of the active coils and conducting wall for RWM stabilisation in ASDEX Upgrade [19]. Prototypical for the application in a stationary high-performance H-mode scenario lim- 


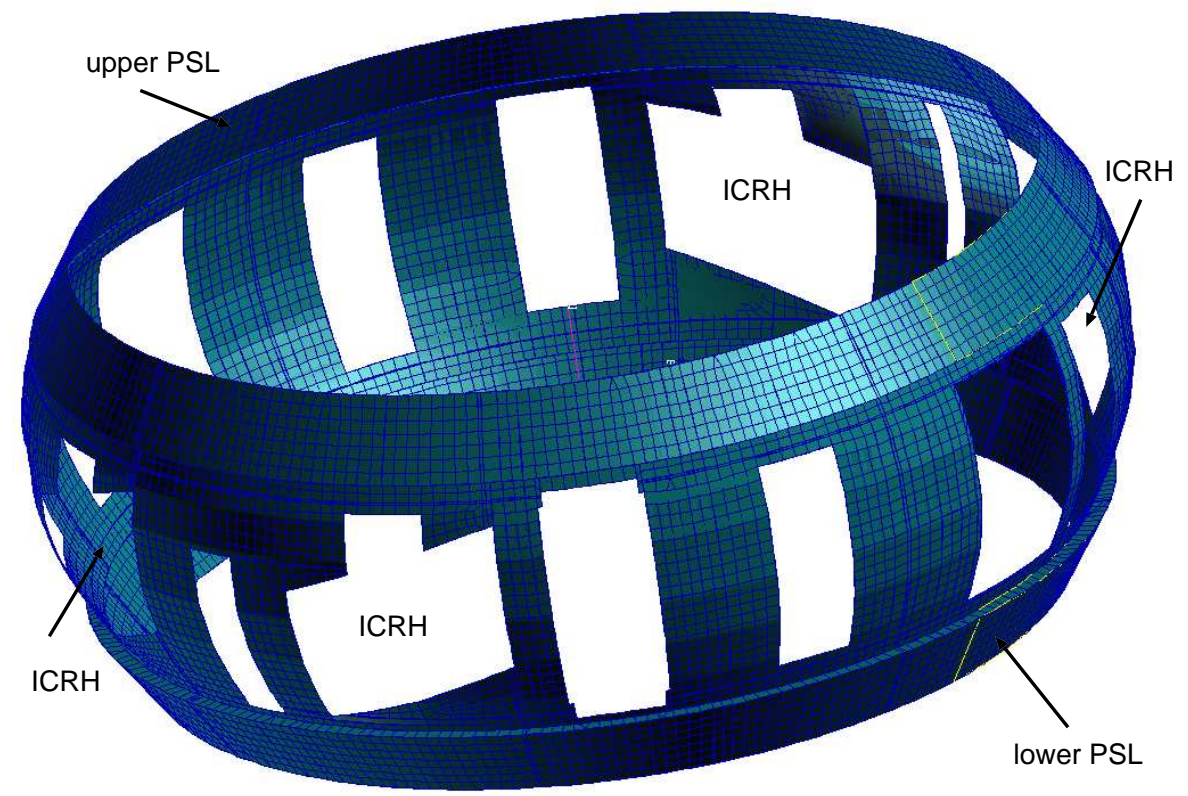

Fig. 6. Finite element model of the conducting wall, including the existing passive stabilising loop.

ited by the RWM, a shaped equilibrium with broad pressure profile $(<\beta\rangle_{V}=$ $\left.2.5 \%, \beta_{N}=2.62\right)$ and broad, non-monotonous $q$ profile $\left(q_{0}=3.3, q_{\min }>2\right.$ at $\rho_{\text {tor }}=0.8$ ) is assumed [17]. For this situation, stability calculations with $n=1 \ldots 2$ and a large range of $m$ show that several eigenmodes are simultaneously unstable [19]. The normalised growth rate $\lambda=\gamma \mu_{0} \sigma d$ ( $\gamma$ : growth rate, $\sigma$ : wall conductivity, $d$ : wall thickness) is found to be $\lambda=7.7 \mathrm{~m}^{-1}, 7.5 \mathrm{~m}^{-1}$ ( $n=1$ dominated modes), $6.24 \mathrm{~m}^{-1}$ and $5.88 \mathrm{~m}^{-1}$ ( $n=2$ dominated modes). In comparison, 2D stability calculations with CASTOR, assuming an axisymmetric contiguous wall, yield a much lower growth rate, $\lambda=1.54 \mathrm{~m}^{-1}(n=1)$ and $\lambda=1.67 \mathrm{~m}^{-1}(n=2)$. With a copper wall of $5 \mathrm{~mm}$ thickness, the corresponding growth rates are between $\gamma=15.6 \mathrm{~s}^{-1}$ and $\gamma=20.5 \mathrm{~s}^{-1}$. The closed loop calculation with OPT IM, using eight toroidally distributed poloidal field sensors to resolve the unstable low $n$ modes, confirms that with feedback on, all eigenmodes are stabilised. The electromagnetic simulations [13] show that at frequencies below $700 \mathrm{~Hz}$ the phase lag between coil current and produced magnetic field at the plasma surface is 25 degrees or less, which (depending on the remaining elements of the control loop) should allow to design the control loop with sufficient phase margin. Further work is devoted to include a more detailed model (transfer function) of the sensors, the controller, power supplies, and power cables in the stability assessment. 


\section{Summary and Conclusion}

The proposed set of $3 \times 8$ in-vessel saddle coils allows flexible perturbation field configurations. For ELM control experiments, $n=4$ resonant magnetic perturbations can be produced (and compared to lower $n$ perturbations) that can show the advantages of high $n$ for core island avoidance even for cases with low plasma rotation. Three poloidally distributed toroidal sets of coils can produce four different phasings for $n=4$, resonant at different $q$, which allows to test the importance of a resonance for complete ELM suppression. For $n=3$, the spatial phase can be varied quasi-continuously. This can be used to rotate toroidally the perturbation field with respect to the sightlines of the edge diagnostics. In addition, the toroidal phase can be "detuned" from a resonance, which radially shifts and/or broadens the ergodised layers. These studies will allow to prepare operation of similar coils (internal or external) or ferritic inserts for ELM suppression in ITER. Together with a conducting shell and suitable feedback system, the proposed active coils can stabilise the RWM well above the no-wall limit, a further step in preparation of advanced scenarios towards steady-state operation.

\section{References}

[1] G. Federici, Physica Scripta T124 (2006) 1.

[2] A. Loarte, G. Saibene, R. Sartori, D. Campbell, M. Bécoulet, L. Horton, et al., Plasma Phys. Controlled Fusion 45 (2003) 1549.

[3] P. T. Lang, G. D. Conway, T. Eich, L. Fattorini, O. Gruber, S. Günter, et al., Nucl. Fusion 44 (2004) 665.

[4] J. Stober, M. Maraschek, G. D. Conway, O. Gruber, A. Herrmann, A. C. C. Sips, et al., Nucl. Fusion 41 (2001) 1123.

[5] Y. Kamada, T. Oikawa, L. Lao, T. Takizuka, T. Hatae, A. Isayama, et al., Plasma Phys. Controlled Fusion 42 (2000) A247.

[6] N. Oyama, P. Gohil, L. D. Horton, A. E. Hubbard, J. W. Hughes, Y. Kamada, et al., Plasma Phys. Controlled Fusion 48 (2006) A171.

[7] K. H. Burrell, M. E. Austin, D. E. Brennan, J. C. DeBoo, E. J. Doyle, P. Gohil, et al., Plasma Phys. Controlled Fusion 44 (2002) A253.

[8] W. Suttrop, V. Hynönen, T. Kurki-Suonio, P. T. Lang, M. Maraschek, R. Neu, et al., Nucl. Fusion 45 (2005) 721.

[9] T. C. Hender, R. Fitzpatrick, A. W. Morris, P. G. Carolan, R. D. Durst, T. Edlington, et al., Nucl. Fusion 32 (1992) 2091. 
[10] T. E. Evans, R. A. Moyer, P. R. Thomas, J. G. Watkins, T. H. Osborne, J. A. Boedo, et al., Phys. Rev. Lett. 92 (2004) 235003.

[11] K. H. Burrell, T. E. Evans, E. J. Doyle, M. E. Fenstermacher, R. J. Groebner, A. W. Leonard, et al., Plasma Phys. Controlled Fusion 47 (2005) B37.

[12] T. Vierle, B. Streibl, M. Rott, U. Seidel, A. Herrmann, O. Neubauer, et al., Design and stress analysis of in-vessel saddle coils for MHD control in ASDEX Upgrade, this conference.

[13] M. Rott, U. Seidel, B. Streibl, W. Suttrop, T. Vierle, and the ASDEX Upgrade Team, Electro-magnetic modeling of the planned active in-vessel coils for ASDEX Upgrade, this conference.

[14] K. H. Finken, A. Abdullaev, W. Biel, M. F. M. de Bock, S. Brezinsek, C. Busch, et al., Contrib. Plasma Phys. 46 (2006) 515.

[15] B. V. Chirikov, Phys. Rep. 52 (1979) 263.

[16] P. Merkel and M. Sempf, in IAEA Fusion Energy Conference 2006, Chengdu, China, paper TH/P3-8, 2006.

[17] E. Strumberger, P. Merkel, M. Sempf, and S. Günter, Phys. Plasmas 15 (2008) 056110.

[18] M. Sempf, P. Merkel, E. Strumberger, and S. Günter, in Europhysics Conference Abstracts (CD-ROM), Proc. of the 35th EPS Conference on Controlled Fusion and Plasma Physics, Hersonissos, Greece, 2008, page P1.077, Geneva, 2008, EPS.

[19] S. Günter, P. Lauber, M. Sempf, E. Strumberger, D. Tekle, and K. Lackner, Plasma Phys. Controlled Fusion 50 (2008) 124004. 